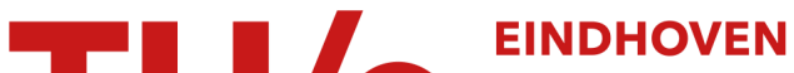 \\ UNIVERSITY OF \\ TECHNOLOGY
}

\section{Redistribution of fast ions during sawtooth reconnection}

Citation for published version (APA):

Jaulmes, F. F. E., Westerhof, E., \& Blank, de, H. J. (2014). Redistribution of fast ions during sawtooth reconnection. Nuclear Fusion, 54, 104013-1/12. https://doi.org/10.1088/0029-5515/54/10/104013

DOI:

10.1088/0029-5515/54/10/104013

Document status and date:

Published: 01/01/2014

\section{Document Version:}

Publisher's PDF, also known as Version of Record (includes final page, issue and volume numbers)

\section{Please check the document version of this publication:}

- A submitted manuscript is the version of the article upon submission and before peer-review. There can be important differences between the submitted version and the official published version of record. People interested in the research are advised to contact the author for the final version of the publication, or visit the $\mathrm{DOI}$ to the publisher's website.

- The final author version and the galley proof are versions of the publication after peer review.

- The final published version features the final layout of the paper including the volume, issue and page numbers.

Link to publication

\section{General rights}

Copyright and moral rights for the publications made accessible in the public portal are retained by the authors and/or other copyright owners and it is a condition of accessing publications that users recognise and abide by the legal requirements associated with these rights.

- Users may download and print one copy of any publication from the public portal for the purpose of private study or research.

- You may not further distribute the material or use it for any profit-making activity or commercial gain

- You may freely distribute the URL identifying the publication in the public portal.

If the publication is distributed under the terms of Article $25 \mathrm{fa}$ of the Dutch Copyright Act, indicated by the "Taverne" license above, please follow below link for the End User Agreement:

www.tue.nl/taverne

Take down policy

If you believe that this document breaches copyright please contact us at:

openaccess@tue.nl

providing details and we will investigate your claim. 
Redistribution of fast ions during sawtooth reconnection

This content has been downloaded from IOPscience. Please scroll down to see the full text. 2014 Nucl. Fusion 54104013

(http://iopscience.iop.org/0029-5515/54/10/104013)

View the table of contents for this issue, or go to the journal homepage for more

Download details:

IP Address: 131.155.151.137

This content was downloaded on 20/01/2015 at $11: 49$

Please note that terms and conditions apply. 


\title{
Redistribution of fast ions during sawtooth reconnection
}

\author{
F. Jaulmes, E. Westerhof and H.J. de Blank \\ FOM Institute DIFFER Dutch Institute For Fundamental Energy Research, \\ Association EURATOM-FOM, Nieuwegein, The Netherlands \\ E-mail: F.Jaulmes@differ.nl
}

Received 15 November 2013, revised 29 July 2014

Accepted for publication 5 August 2014

Published 1 October 2014

\begin{abstract}
In a tokamak-based fusion power plant, possible scenarios may include regulated sawtooth oscillations to remove thermalized helium from the core of the plasma. During a sawtooth crash, the helium ash and other impurities trapped in the core are driven by the instability to an outer region. However, in a fusion plasma, high energy ions will represent a significant population. We thus study the behaviour of these energetic particles during a sawtooth. This paper presents the modelling of the redistribution of fast ions during a sawtooth reconnection event in a tokamak plasma. Along the lines of the model for the evolution of the flux surfaces during a sawtooth collapse described in Ya.I. Kolesnichenko and Yu.V. Yakovenko 1996 Nucl. Fusion 36 159, we have built a time-dependent electromagnetic model of a sawtooth reconnection. The trajectories of the ions are described by a complete gyro-orbit integration. The fast particles were evolved from specific initial parameters (given energy and uniform spread in pitch) or distributed initially according to a slowing-down distribution created by fusion reactions. Our modelling is used to understand the main equilibrium parameters driving the motions during the collapse and to determine the evolution of the distribution function of energetic ions when different geometries of reconnection are considered.
\end{abstract}

Keywords: sawtooth, fast ions, particle motion, tokamak, impurities, alpha-particles

(Some figures may appear in colour only in the online journal)

\section{Introduction}

In a tokamak-based fusion power plant, thermal helium and impurities will tend to accumulate in the core region of the plasma [1]. By moving the bulk of the core plasma to an outer region, the sawtooth instability has the unique feature to flush impurities retained in the core outward. Active control of the sawtooth period and amplitude provides the capability to optimize this desired consequence of the sawtooth. The motivation behind this work is to build scenarii where a controlled version of the instability is used to improve the performances of the fusion plasma.

In fusion devices, the high energy ions represent a significant part of the plasma energy and of the current drive: we are thus interested in the consequences of the sawtooth on these fast ions. Understanding of the effects of the sawtooth collapse on the fast ion population is of importance for the development of a coherent control strategy of the sawtooth cycle that would take into account the production of alphaparticles and the deposition of energy and current from the auxiliary heating systems. The redistribution of the fast ions will also change their interaction with the background plasma and possibly destabilize toroidal Alfvén eigenmodes (TAEs), neoclassical tearing modes (NTMs) or energetic particle modes. We would like to avoid these side-effects.
Many experimental and theoretical studies have recently been dedicated to the control of sawtooth oscillations [2]. A sawtooth crash is triggered when the $m=1, n=1$ resistive internal kink mode is destabilized [3], where $m$ and $n$ are the poloidal and toroidal mode numbers, respectively. This instability corresponds to an almost rigid displacement of the plasma core with $m=1, n=1$ helicity. All heating and/or current drive methods like neutral beam injection (NBI), ion cyclotron resonance heating (ICRH) and electron cyclotron resonance heating $(\mathrm{ECRH})$ can alter the trigger of the sawtooth, either by modifying the magnetic shear $s=(r / q)(\mathrm{d} q / \mathrm{d} r)$ at the resonant surface with safety factor $q=1$ or by acting on the local distribution of the fast ions [4].

In this paper, we investigate the influence of the sawtooth on the fast ions when different initial magnetic geometries of the equilibrium are considered. Since we are interested in sawteeth in plasmas that are heated and stabilized by the fusion born alpha-particles, we consider, as described in [5], that the plasma is operated in the stable region between sawtooth and fishbone instabilities. We develop a numerical model of the sawtooth collapse based on the original full reconnection pattern suggested by Kadomtsev [6] with the dynamical evolution of the electromagnetic fields during the collapse as introduced by Kolesnichenko and Yakovenko in [7]. We then calculate the full particle orbits in these time 
evolving fields in order to analyze the effect of a sawtooth collapse on different populations of thermal and energetic ions. Compared with previous work, we use realistic magnetic equilibria and the particle motions are fully described along their orbits, allowing the modelling to account for the effect of the Larmor orbits and the orbit widths of the ions. This is applied to understand the effect of the collapse on different orbit categories (passing or trapped), at different approximate radial regions (core or around $q=1$ ), and for a large range of kinetic energies (up to $3.5 \mathrm{MeV}$ ).

This paper is structured as follows. The model for the dynamical evolution of the electromagnetic field during a sawtooth collapse is discussed in section 2. In section 3, the algorithm used for the numerical integration of the particle orbits is presented together with a validation of the implementation. A comprehensive analysis of the effect of the sawtooth collapse on the different kinds of particles is then presented in section 4. The main emphasis is on the degree to which the different particles follow the redistribution of the magnetic flux during the collapse. All calculations are performed starting from JET-like equilibria. Section 5 then presents the effect of a sawtooth collapse on a slowing down distribution of fusion produced alpha-particles. Finally, section 6 summarizes the conclusions of this study.

\section{Dynamical modelling of the sawtooth collapse}

\subsection{Pre-collapse equilibrium and resonant surface}

As a starting point for this work, we have used tokamak equilibria as obtained from the equilibrium code FINESSE [8]. The typical parameters considered are those of a JETlike configuration, where $P_{0} \sim 1.1 \times 10^{5} \mathrm{~Pa}$ is the pressure on the magnetic axis, $I_{\mathrm{P}} \sim 2.5 \mathrm{MA}$ is the global toroidal plasma current and $B_{\text {axis }} \sim 3.3 \mathrm{~T}$ is the total field on axis. The simulations were performed with the current in the same positive direction as that of the toroidal field. Co-passing particles are considered orbiting in the direction of the toroidal field. Reversing the current direction would exchange the behaviour of the co- and counter- passing particles. The Shafranov shift is $\sim 0.15 \mathrm{~m}$ for $\beta=\left(2 \mu_{0}\langle P\rangle\right) /\left\langle B^{2}\right\rangle \sim 1.1 \%$ (where $\langle P\rangle$ is the volume averaged pressure of the plasma, $B$ is the magnetic field). We obtain from the equilibrium code the total field with its poloidal and toroidal components: $\boldsymbol{B}=\boldsymbol{B}_{\text {pol }}+\boldsymbol{B}_{\varphi}$. The poloidal field is related to a flux $\psi$, that is the (divided by $2 \pi$ ) poloidal magnetic flux, so that $\boldsymbol{B}_{\mathrm{pol}}=(1 / R)\left(\boldsymbol{e}_{\varphi} \times \nabla \psi\right)$. The equilibrium code also gives us a mesh in the poloidal plane: it provides the horizontal coordinate $X(\psi, \theta)=R(\psi, \theta)-R_{0}$ and the vertical one $Z(\psi, \theta)$. Here $R_{0}=\left(R_{\max }+R_{\min }\right) / 2$ is the center of a Fourier decomposition in geometric poloidal angle (see [8]) of the last closed flux surface ( $R_{\max }$ and $R_{\min }$ being respectively the maximum and minimum values of the major radius along this flux surface) and $\theta$ is a straight field line poloidal angle. On a given flux surface, the safety factor $q=\mathrm{d} \varphi / \mathrm{d} \theta$ then describes the pitch of a field line ( $\varphi$ being the toroidal angle). Since we consider particles evolving close to $q=1$, it is of interest to consider their position with respect to this helicity by using the helical angle $\omega=\theta-\varphi$. For convenience, we use a length index $r$ (effective radial position) to label flux surfaces. It is defined as
$r=\sqrt{\mathcal{S}(\psi) / \pi}$ where $\mathcal{S}(\psi)$ is the area of the surface enclosed by the poloidal contour of the flux surface $\psi$.

Let $\boldsymbol{B}_{\mathrm{H}}$ be the magnetic field that the plasma would have in case of a uniform $q=1$ helicity. The equilibrium configuration, and in particular the equilibrium poloidal field $\boldsymbol{B}_{\mathrm{pol}}$, allows us to give a description of this reference helical field: $\boldsymbol{B}_{\mathrm{H}}=q \boldsymbol{B}_{\mathrm{pol}}+B_{\varphi} \boldsymbol{e}_{\varphi}$ ( $\boldsymbol{e}_{\varphi}$ being a toroidal unit vector). We can relate this field to a poloidal flux $\psi_{\mathrm{H}}$, so that: $q \boldsymbol{B}_{\mathrm{pol}}=$ $(1 / R)\left(e_{\varphi} \times \nabla \psi_{\mathrm{H}}\right)$.

We then define an auxiliary field $\boldsymbol{B}_{*}=\boldsymbol{B}-\boldsymbol{B}_{\mathrm{H}}=$ $(1-q) \boldsymbol{B}_{\mathrm{pol}}$ that describes the deviation of the equilibrium field from the $q=1$ helicity. We finally introduce the function $\psi_{*}$, poloidal flux of the auxiliary poloidal field $\boldsymbol{B}_{*}$ (auxiliary flux or so-called helical flux [9]). These are related by $\boldsymbol{B}_{*}=(1 / R)\left(\boldsymbol{e}_{\varphi} \times \nabla \psi_{*}\right)$. In our calculations, we first derive the auxiliary flux in the equilibrium by integrating according to our definitions:

$$
\psi_{*}(t=0)=\psi_{* \mathrm{eq}}(\psi)=\int_{\psi_{0}}^{\psi}(1-q) \mathrm{d} \psi .
$$

All dynamics during the collapse is simply described by the evolution of $\psi_{*}(t)$. Under the assumption that the shaping of the plasma is unaffected by the reconnection, $\psi_{*}$ is again a function of $\psi$ only after the sawtooth.

An illustration of reconnection occurring during a sawtooth collapse according to Kadomtsev [6] is given on figure 1 . Surfaces that have identical auxiliary flux $\psi_{*}$ values on both sides of the separatrix (effective radial position $r_{x}$, see figure 2) reconnect during the crash. This results in an incompressible flow of plasma. The reconnection actually takes place in a narrow layer where a strong current appears. The mixing radius $r_{\text {mix }}$ is the post-crash position of the separatrix (where, after the collapse, a discontinuity in $q$ is present).

\subsection{Dynamics of the sawtooth collapse}

The initial magnetohydrodynamics (MHD) instability ( $m=1$, $n=1$ kink motion) may be triggered by the properties of the fast ion distributions or the current density profile as affected by current drive. We assume it is accompanied in its nonlinear phase by complete reconnection of the auxiliary magnetic field around the $q=1$ resonant flux surface, finally resulting in a new axi-symmetric state of the plasma.

Our modelling is based on the one from Kolesnichenko et al in [7]. In this model, $(r, \omega=\theta-\varphi, \varphi)$ are the helical coordinates with the $\varphi$ angle being the toroidal coordinate. The entire collapse consists of two phases: a first phase (which we will call the crash phase) in which the auxiliary field is reconnected while the core or the plasma is moved radially outward as displayed on figure 2. This phase ends when the core reaches the mixing radius and the reconnection is completed, but leaves the plasma in a helically perturbed state. In a subsequent phase the axisymmetry is restored. These two phases constitute the entire collapse.

The evolving flux surfaces are mathematically described in the $(r, \omega)$ coordinates by the equations given in [7]. A shape parameter $\alpha$ is introduced that determines a continuous change of the shape of the flux surfaces. $\alpha$ evolves from 0 at the beginning to $\operatorname{atan}(2)$ at the end of the crash (first phase) when 

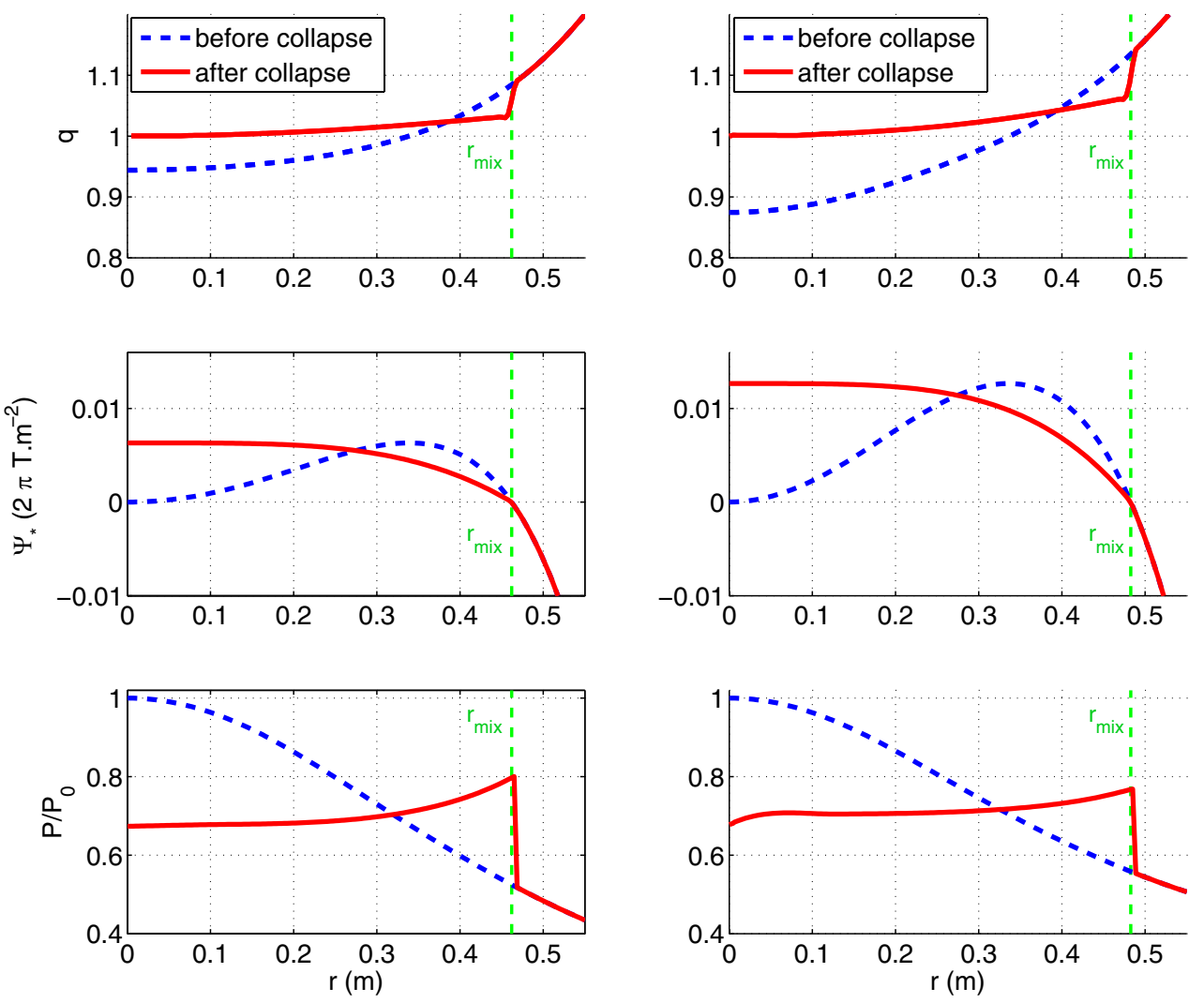

Figure 1. Radial profiles, before and after the collapse, of the auxiliary flux (middle plots) and corresponding safety factor profiles (top plots) and pressure profiles (lower plots) according to Kadomtsev theory. Two different safety factor profiles are considered: one with low shear (left) and one with higher shear (right). In our modelling, the discontinuity of the magnetic field (and thus in $q$ ) appears during the second phase of the collapse.

reconnection is complete. Then it increases up to $\alpha=\pi$ when axi-symmetry of the field is restored. The temporal evolution is obtained by assuming a linear evolution of $\alpha$ with time:

$$
\frac{t}{\tau_{\mathrm{cr}}}=\frac{\alpha}{\operatorname{atan}(2)} ; \quad 0 \leqslant \alpha \leqslant \pi
$$

We thus have $\alpha(t)=\left(\operatorname{atan}(2) / \tau_{\text {cr }}\right) t$ where the crash time $\tau_{\text {cr }}$ is the duration of the first phase. This first phase is the phase with the highest drifts and its duration is the one usually obtained from experimental measurements.

We define $\xi$ as the magnitude of the displacement of the original magnetic axis. The complete reconnection of the flux surfaces inside the $q=1$ surface implies that the process will stop at a given flux surface position, the mixing radius $r_{\text {mix }}$, which defines the extension of the zone where the electromagnetic fields are perturbed. The radius of the external circle of the separatrix is at position $r_{x}(t)$. The displacement of the center of the plasma column is evolved during the time of the crash according to: $\xi(t)=(1 / 2) r_{x}(t) \tan (\alpha(t))$ for $0 \leqslant \tan (\alpha(t)) \leqslant 2$ and $\xi(t)=r_{\text {mix }}$ for $\tan (\alpha(t))>2$.

This modelling is based on ideal MHD and thus allows to set up the crash and collapse times arbitrarily. The reconnection dynamics can be either mostly resistive (see [9]) or collisionless (see [10]). Since we assume that we operate in conditions of a DT experiment in JET, we may use the collisionless estimate for the complete reconnection time:

$$
\tau_{\mathrm{cr}} \simeq \frac{\tau_{A}}{\rho_{s} q^{\prime}} \quad \text { with } \quad \rho_{s}=\frac{\sqrt{\left(T_{\mathrm{e}}+T_{\mathrm{i}}\right) / m_{\mathrm{i}}}}{\Omega_{c i}} .
$$

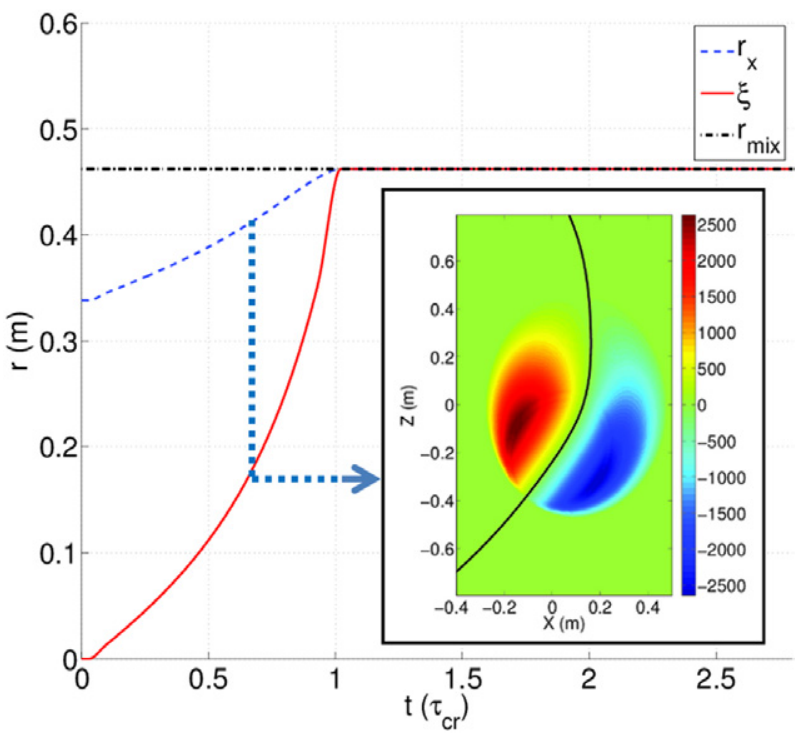

Figure 2. Evolution of the position of the core $(\xi)$ and of the separatrix $\left(r_{x}\right)$ during the collapse of the low shear scenario (left graphs) of figure 1 . The inset illustrates the calculation (equation (6)) of the electric potential at one instant; the full line indicates the reference surface $\omega=0$ and $\omega=\pi$ where $\Phi=0$.

Here $\tau_{A} \sim R / v_{A}$ is an Alfvén time and $\Omega_{c i}=\left(Z_{\mathrm{ie}}\right) B_{0} / m_{\mathrm{i}}$. The crash times that we consider in this study are $\tau_{\mathrm{cr}} \sim 144 \mu \mathrm{s}$ in section 4 and $\tau_{\mathrm{cr}} \sim 90 \mu \mathrm{s}$ in section 5. The total collapse is assumed to last about $2.78 \tau_{\mathrm{cr}}$. 
Using the shapes of $\psi_{*}$ from the model in [7] and the area conservation principle from [6] we thus can build a complete phenomenological 'movie' of the collapse described by the perturbed magnetic potential $\psi_{*}(r, \omega, t)$.

\subsection{Magnetic field and electric field}

We get the overall magnetic field by using $\boldsymbol{B}=\boldsymbol{B}_{\mathrm{H}}+\left(\boldsymbol{e}_{\varphi} \times\right.$ $\left.\nabla \psi_{*}\right) / R$. We may write the electric fields associated with this magnetic field evolution as:

$$
\boldsymbol{E}=\frac{\dot{\psi_{*}}}{R} \boldsymbol{e}_{\varphi}-\nabla \Phi
$$

Most of the plasma during the collapse can be considered frozen in the magnetic field and described by ideal MHD, except for the small region where the reconnection of the field lines occurs and where a current is flowing (moving 'reconnecting zone' where the inner and outer separatrix touch each other). We now assume that the electric potential is constant along the helical direction so that $\boldsymbol{\nabla} \Phi \cdot \boldsymbol{B}_{\mathrm{H}}=0$. Ideal MHD yields $\boldsymbol{E} \cdot \boldsymbol{B}=0$ and, using (4), we obtain a characteristic equation:

$$
\frac{\partial \Phi}{\partial X} B_{* X}+\frac{\partial \Phi}{\partial Z} B_{* Z}=\dot{\psi}_{*} B_{\varphi} / R .
$$

We can integrate (5) along a contour where $\psi_{*}$ is constant. Denoting $\mathrm{d} l_{*}$ the length of a contour element, this yields for the electric potential the expression:

$$
\Phi=\int_{\psi_{*} \text { contour }} \frac{B_{\varphi} \dot{\psi_{*}}}{R B_{*}} \mathrm{~d} l_{*}+\Phi_{0}(\psi) .
$$

The constant may be a flux surface quantity and should be related to a rotation-induced equilibrium profile of the electrostatic potential. However, since it has been shown previously [11] that plasma rotation has little impact on the fast ions during a collapse, we have decided to arbitrarily set the potential to zero for the angular position corresponding to a specific 'helical' surface: we set $\Phi=0$ for the positions $\omega=0$ and $\omega=\pi$ (see figure 2).

We integrate (6) in the poloidal plane for given values of toroidal position $\varphi$. After transforming $\theta$ to $\omega$ and averaging over several $\varphi$ values to increase numerical accuracy, this allows us to express $\Phi$ as a function of $r$ and $\omega$. The total electric field is then expressed in tokamak cylindrical coordinates by using equation (4).

An illustration of the radial motions resulting from the corresponding electrostatic drift $\boldsymbol{v}_{\mathrm{E}}$ is given on figure 3 at $t / \tau_{\mathrm{cr}} \simeq 0.86$. To understand how particles may escape the radial redistribution, one should consider that a particle that would be freely circulating in the $\omega$ direction and does exactly one turn during the crash while staying at a constant flux surface position (constant $r$ ) would almost average out the radial component of the $\boldsymbol{E} \times \boldsymbol{B}$ drift ( $\left.v_{\mathrm{Er}}\right)$ to zero: this is shown on the left part of figure 4 .

\section{Motion of fast ions}

\subsection{Description of the particle motion}

In a plasma, it is convenient to decompose the speed as $\boldsymbol{v}=\boldsymbol{v}_{\perp}+\boldsymbol{v}_{\|}$, where the $\|$component is obtained by projection
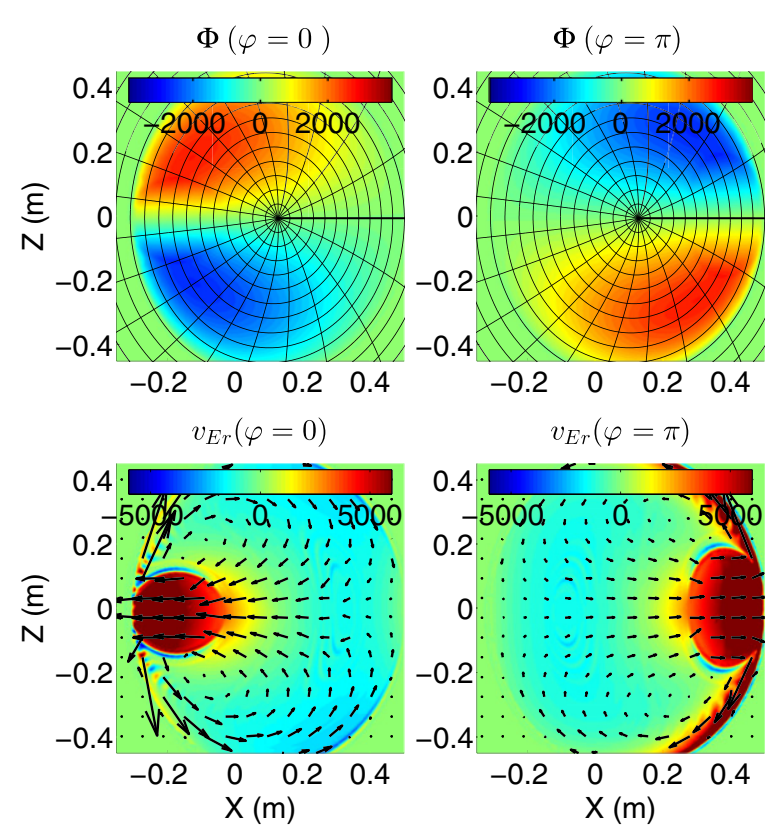

Figure 3. The upper figures show the projection of the electric potential $\Phi$ (in V) in the poloidal plane at time $t / \tau_{\mathrm{cr}} \simeq 0.86$. The lower figures represent the direction of the electrostatic drift $\boldsymbol{v}_{\mathrm{E}}$ and the color scale its intensity along the outward radial direction (in $\mathrm{ms}^{-1}$ ). The particles that follow mainly $\boldsymbol{v}_{\mathrm{E}}$ stay attached to the field and thus are redistributed. The left figures are at position $\varphi=0$ while the right ones are for $\varphi=\pi$.

along the magnetic field line. For the study of motions in a tokamak, we also use below the decomposition $\boldsymbol{v}=\boldsymbol{v}_{p o l}+\boldsymbol{v}_{\varphi}$ where $v_{\text {pol }}$ is the component of the particle speed in the poloidal plane $\left(\boldsymbol{v}_{\mathrm{pol}}=v_{X} \boldsymbol{e}_{\mathrm{X}}+v_{Z} \boldsymbol{e}_{\mathrm{Z}}\right)$. We use tokamak cylindrical coordinates $\left(X=R-R_{0}, Z, \varphi\right)$, so that $e_{\varphi}=e_{X} \times e_{Z} \cdot e_{X}$ and $e_{\mathrm{Z}}$ are the orthogonal unit vectors in the poloidal plane. The position of a particle is defined as $r=R e_{\mathrm{X}}+Z e_{\mathrm{Z}}$. In these coordinates, the acceleration can be expressed as: $\frac{\mathrm{d}^{2} r}{\mathrm{~d} t^{2}}=\left(\ddot{R}-R \dot{\varphi}^{2}\right) \boldsymbol{e}_{\mathrm{X}}+(R \ddot{\varphi}+2 \dot{R} \dot{\varphi}) \boldsymbol{e}_{\varphi}+\ddot{Z} \boldsymbol{e}_{\mathrm{Z}}$. For an ion of charge $\left(Z_{\mathrm{i}} e\right)$, we project the terms of the momentum equation along the toroidal direction:

$$
\begin{gathered}
\left(\frac{\mathrm{d}^{2} \boldsymbol{r}}{\mathrm{d} t^{2}}\right)_{\varphi}=\frac{1}{R} \frac{\mathrm{d}}{\mathrm{d} t}\left(R v_{\varphi}\right), \quad E_{\varphi}=\frac{1}{R}\left(\frac{\partial \psi}{\partial t}-\frac{\partial \Phi}{\partial \varphi}\right) \\
{[\boldsymbol{v} \times \boldsymbol{B}]_{\varphi}=\frac{1}{R}\left(\boldsymbol{v}_{\mathrm{pol}} \cdot \nabla \psi\right) .}
\end{gathered}
$$

Furthermore, since:

$$
\frac{\partial \psi}{\partial t}+\boldsymbol{v}_{\mathrm{pol}} \cdot \nabla \psi=\frac{\mathrm{d} \psi}{\mathrm{d} t}-v_{\varphi} \frac{1}{R} \frac{\partial \psi}{\partial \varphi},
$$

we may combine these results to rewrite the momentum equation of a particle:

$$
\frac{1}{R} \frac{\mathrm{d}}{\mathrm{d} t}\left(R v_{\varphi}\right)=\frac{Z_{\mathrm{i}} e}{m} \frac{1}{R}\left(\frac{\mathrm{d} \psi}{\mathrm{d} t}-\frac{\partial \Phi}{\partial \varphi}-\frac{v_{\varphi}}{R} \frac{\partial \psi}{\partial \varphi}\right),
$$

and thus:

$$
\frac{\mathrm{d}}{\mathrm{d} t}\left(R m \mathrm{v}_{\varphi}-\left(Z_{\mathrm{i}} e\right) \psi\right)=-\left(Z_{\mathrm{i}} e\right)\left[\frac{\partial \Phi}{\partial \varphi}+\frac{v_{\varphi}}{R} \frac{\partial \psi}{\partial \varphi}\right] .
$$

We may note that a consequence of this result is that, in an axi-symmetric configuration, the quantity on the LHS of 

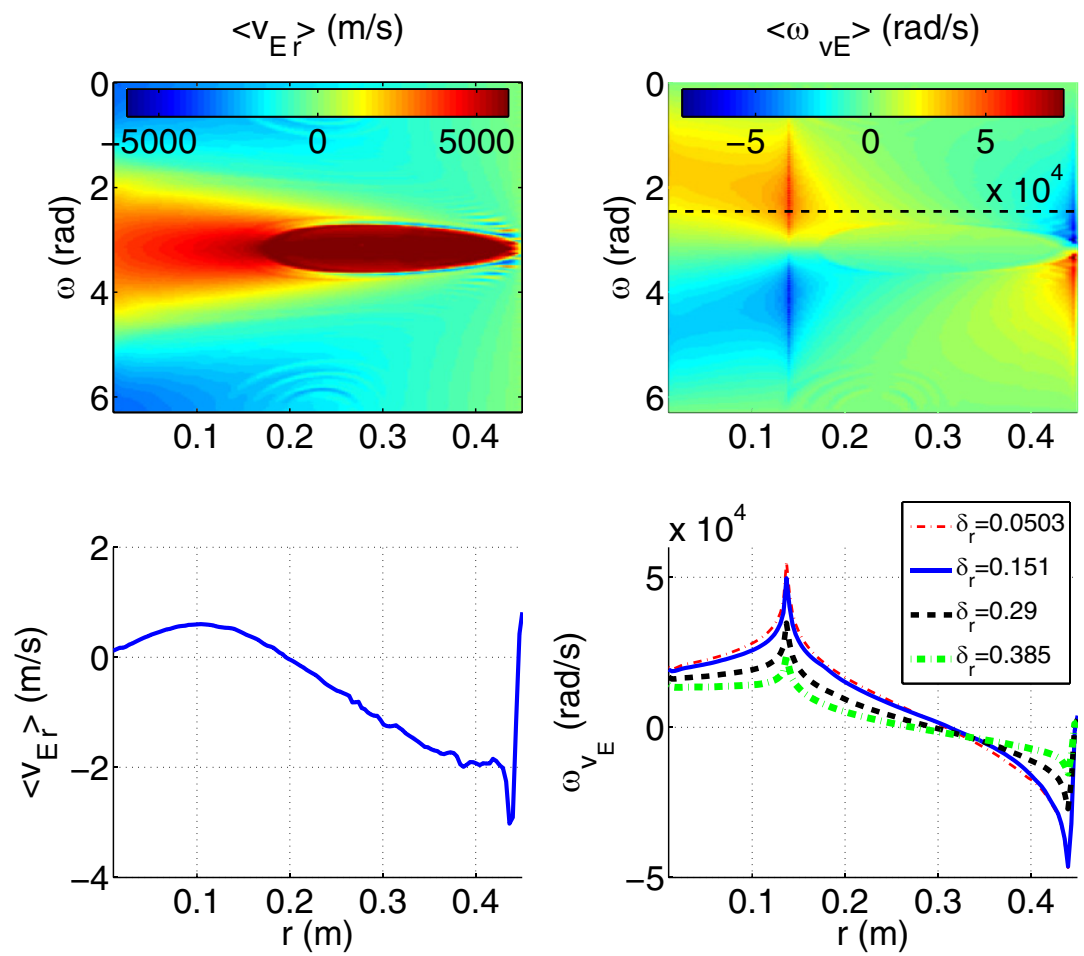

Figure 4. Toroidally averaged values of radial $\left(v_{\mathrm{Er}}\right)$ and helical $\left(\omega_{v E}\right)$ electrostatic drifts at time $t / \tau_{\mathrm{cr}} \simeq 0.86$. Upper left: estimate at one instant of $v_{\mathrm{Er}}$ in the $(r, \omega)$ coordinates. Lower left: when averaging along all $\omega$ positions, the resulting radial drift is almost zero. Upper right: an estimate in the $(r, \omega)$ coordinates of $\omega_{v_{E}}$ at one instant during the collapse. The lower right figure shows that, for a given helical position, when averaging along several radial positions, $\omega_{v_{E}}$ may be lowered for larger orbit widths (the values are given here at $\omega \simeq 2.45$ ).

equation (7), $p_{\varphi}=R m \mathrm{v}_{\varphi}-\left(Z_{\mathrm{i}} e\right) \psi$ is conserved. $p_{\varphi}$ is the toroidal canonical angular momentum [12], that we can use as an estimate of the average flux surface position of a fast particle with given pitch and energy. On the plots given in this paper, we have divided the various values of $p_{\varphi}$ by the elementary charge $e$ for simplicity. Thus the values plotted are actually that of $p_{\varphi} / e$ (in $2 \pi \mathrm{Wb}$ ) except on figure 8 where the values in the top plot have been normalized to the thermal redistribution. The graphics only mention $p_{\varphi}$ on the axes (without units) for clarity.

Since the magnetic field does not exert any work on the particle, the evolution of the kinetic energy $\mathcal{E}_{\text {kin }}$ may be described as: $\dot{\mathcal{E}}_{\text {kin }}=\left(Z_{\mathrm{i}} e\right) \boldsymbol{v} \cdot \boldsymbol{E}$. This expression can be used to express the rate of change of the total energy $\mathcal{E}=\mathcal{E}_{\text {kin }}+\mathcal{E}_{\text {pot }}$ of the particle in terms of the variations of the fields by noting that:

$\frac{\mathrm{d} \mathcal{E}}{\mathrm{d} t}=\frac{\mathrm{d} \mathcal{E}_{\text {kin }}}{\mathrm{d} t}+\left(Z_{\mathrm{i}} e\right) \frac{\mathrm{d} \Phi}{\mathrm{d} t}=\left(Z_{\mathrm{i}} e\right) \boldsymbol{v} \cdot \boldsymbol{E}+\left(Z_{\mathrm{i}} e\right)\left(\boldsymbol{v} \cdot \nabla \Phi+\frac{\partial \Phi}{\partial t}\right)$.

Then, using equation (4) of our modelling, we may write:

$$
\frac{\mathrm{d} \mathcal{E}}{\mathrm{d} t}=\left(Z_{\mathrm{i}} e\right)\left[\frac{v_{\varphi}}{R}\left(\frac{\partial \psi}{\partial t}\right)+\left(\frac{\partial \Phi}{\partial t}\right)\right] .
$$

The relative change in kinetic energy $\mathcal{E}_{\text {kin }}$ proves to be small during the collapse for high energy ions. Finally, a quantity of interest is the magnetic moment of a particle, defined as the magnetic flux through the current ring of its Larmor orbit: it can be related to the perpendicular energy of the particle so that: $\mu=m v_{\perp}^{2} /(2 B)$ and $\mathcal{E}_{\text {kin }}=\mu B+\mathcal{E}_{\|}$. We will also be using the variable $\lambda_{0}=\mu B_{\text {axis }} / \mathcal{E}_{\text {kin }}$, an approximate constant of motion related to the pitch angle of the particle ( $B_{\text {axis }}$ being the total magnetic field on the shifted axis in the equilibria).

\subsection{Algorithm: full-orbit solution in cylindrical coordinates}

Evolving the momentum equation in time can be done in a rather simple way by first integrating to obtain the speeds at half time step. We need to use the velocity vector $\boldsymbol{v}=$ $v_{X} e_{X}+v_{Z} e_{Z}+v_{\varphi} \boldsymbol{e}_{\varphi}$ to integrate and get the position. However, in cylindrical coordinates, the derivative of the velocity vector is written as:

$$
\frac{\mathrm{d} \boldsymbol{v}}{\mathrm{d} t}=\frac{\mathrm{d}\left(v_{X} \boldsymbol{e}_{\mathrm{X}}\right)}{\mathrm{d} t}+\frac{\mathrm{d}\left(v_{Z} \boldsymbol{e}_{\mathrm{Z}}\right)}{\mathrm{d} t}+\frac{\mathrm{d}\left(v_{\varphi} \boldsymbol{e}_{\varphi}\right)}{\mathrm{d} t}=\left(\begin{array}{c}
\dot{v_{X}}-v_{\varphi} \dot{\varphi} \\
\dot{v_{Z}} \\
\dot{v_{\varphi}}+v_{X} \dot{\varphi}
\end{array}\right) .
$$

The momentum equation in these coordinates is thus:

$$
m\left(\begin{array}{c}
\dot{v_{X}} \\
\dot{v_{Z}} \\
\dot{v_{\varphi}}
\end{array}\right)=\left(Z_{\mathrm{i}} e\right)(\boldsymbol{E}+\boldsymbol{v} \times \boldsymbol{B})+m\left(\begin{array}{c}
v_{\varphi} \dot{\varphi} \\
0 \\
-v_{X} \dot{\varphi}
\end{array}\right) .
$$

We then define a corrected magnetic field:

$$
\boldsymbol{B}_{\mathrm{cyl}}=\boldsymbol{B}-\left(\begin{array}{c}
0 \\
\left(m /\left(Z_{\mathrm{i}} e\right)\right) \dot{\varphi} \\
0
\end{array}\right) \quad \text { and } \quad \boldsymbol{b}_{\mathrm{cyl}}=\boldsymbol{B}_{\mathrm{cyl}} / \boldsymbol{B}_{\mathrm{cyl}} .
$$

Using a leap-frog scheme, we then may write:

$$
\boldsymbol{v}^{n+1 / 2}=[\boldsymbol{I}-\boldsymbol{M U}]^{-1}[\boldsymbol{I}+\boldsymbol{M U}] \boldsymbol{v}^{n-1 / 2}
$$

$$
+[\boldsymbol{I}-\boldsymbol{M U}]^{-1} \boldsymbol{E} \frac{Z_{\mathrm{i}} e}{m} \Delta t
$$

with : $\quad[\boldsymbol{I}-\boldsymbol{M U}]^{-1}=\left(\boldsymbol{I}+\mathcal{U}^{2} \boldsymbol{b}_{\text {cyl }} \boldsymbol{b}_{\text {cyl }}^{\top}+\mathcal{U} \boldsymbol{M}\right) /\left(1+\mathcal{U}^{2}\right)$ 

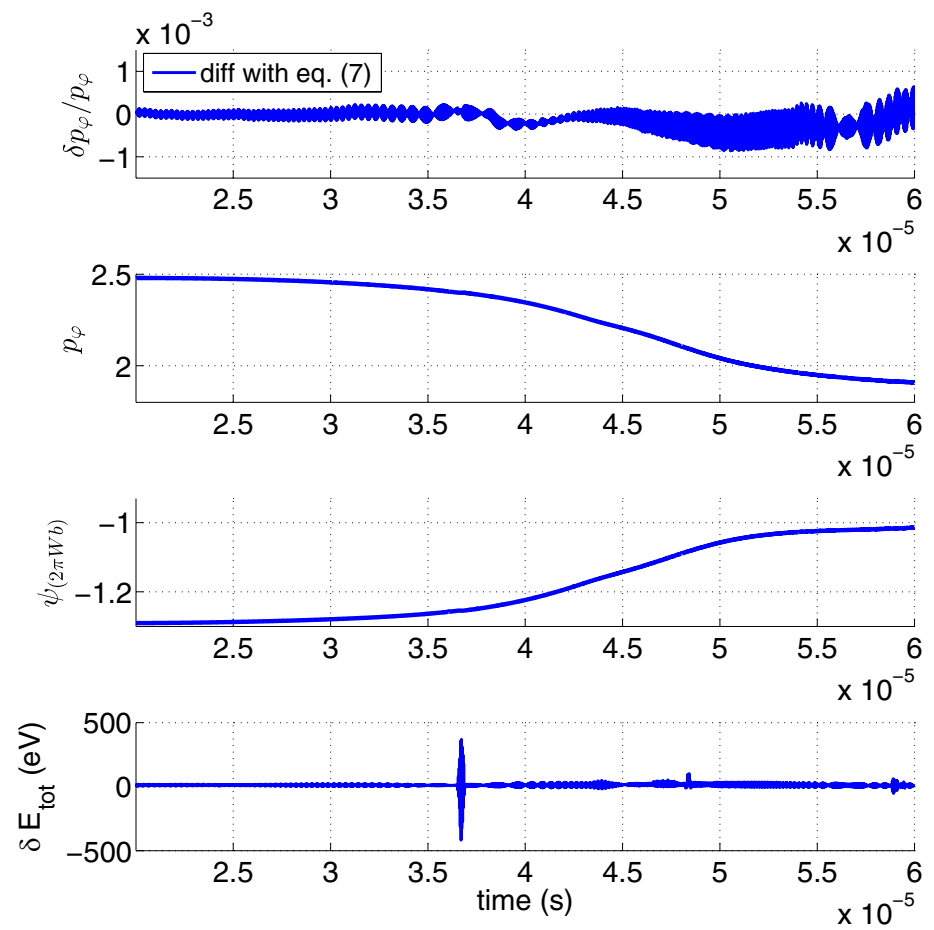

Figure 5. Illustration of the consistency of the evolution of $p_{\varphi}$ for a counter-passing ion of $40 \mathrm{keV}$ (above): the error between the calculated value from the particle position and the one from equation (7) remains below $0.2 \%$. The crash time was $\tau_{\mathrm{cr}} \sim 49 \mu$ s and we see the core expulsion (fast-growing phase of $\xi$ ). The second and third graphs show the change in $p_{\varphi}$ and $\psi$ induced by the change of radial position (outward motion) of the ion. In the lower graph, $\delta E_{\text {tot }}$ represents the difference between integrated value of $\mathcal{E}_{\text {tot }}$ from equation (9) and the value derived from equation (8).

where the fields values on the rhs of (9) are taken at time $n$ and:

$$
\begin{gathered}
\mathcal{U}=\frac{\left(Z_{\mathrm{i}} e\right) B_{\mathrm{cyl}}}{m} \Delta t / 2 \\
M=\frac{1}{B_{\text {cyl }}}\left(\begin{array}{ccc}
0 & B_{\varphi} & -B_{c y l} \\
-B_{\varphi} & 0 & B_{X} \\
B_{c y l Z} & -B_{X} & 0
\end{array}\right) .
\end{gathered}
$$

Unfortunately, this scheme does not have a very good stability to field interpolation errors. Furthermore, to compensate inaccuracies related to our numerical modelling of the electromagnetic fields, we enforce $\boldsymbol{E} \cdot \boldsymbol{B}=0$ for all particles by adjusting the value of the toroidal component of $\boldsymbol{E}: E_{\varphi}=$ $-\left(E_{X} B_{X}+E_{Z} B_{Z}\right) / B_{\varphi}$. To avoid accumulation of errors along the trajectory, it is found useful to correct periodically the speed value according to its amplitude derived from the total energy of the particle. We use equation (8) to periodically rescale the next speed value according to: $\boldsymbol{v}_{\text {next }}=\sqrt{2 \mathcal{E}_{\text {kin }} / m_{\mathrm{i}}}(\boldsymbol{v} / v)$. The algorithm with correction has proved to be fast and efficient both in equilibrium and perturbed fields. The overall set of codes for modelling both fields and particle motions are named EBdyna_go and are validated during collapse simulations by evolving independently $p_{\varphi}$ using equation (7) and comparing the value with the intrinsic one related to the particle speed and position (figure 5).

\subsection{Guiding center drift and different categories of orbits}

The guiding center position is recovered by using: $\boldsymbol{r}_{\mathrm{GC}}=$ $\boldsymbol{r}-\left(m /\left(Z_{\mathrm{i}} e\right)\right)(\boldsymbol{B} \times \boldsymbol{v}) / \boldsymbol{B}^{2}$. The drift of the guiding center of the particles related to the geometry of the magnetic field may be expressed in a low- $\beta$ plasma configuration as: $v_{\mathrm{D}}=$
Table 1. Orbit classification.

\begin{tabular}{lllll}
\hline orbit type & $\sigma_{v}$ & $c_{\mathrm{hfs}}$ & $\sigma_{\mathrm{lfs}}$ & $\sigma_{b}$ \\
\hline co-passing & 1 & 1 & 1 & 0 \\
counter-passing & -1 & 1 & 1 & 0 \\
stagnation LFS & any & 0 & 1 & 0 \\
stagnation HFS & any & 1 & 0 & 0 \\
trapped & any & 0 & 1 & 1 \\
potatoes & any & 1 & 1 & 1 \\
\hline
\end{tabular}

$\left(2 \mathcal{E}_{\text {kin }}-\mu B\right)(b \times \nabla B) /\left(Z_{\mathrm{i}} e B^{2}\right)$. The fast particles will tend to drift across the flux surfaces. We denote with $\delta r$ their orbit width.

We basically consider six categories of particles. We first define $\sigma_{v}$, the sign of $v_{\|}$when the amplitude of the parallel speed is maximum. We also define the parameter $c_{\mathrm{hfs}}$ that is set to one if, at any time during its orbit, the particles is crossing the mid-plane $\left(Z=Z_{\text {axis }}\right.$ ) on the high field side (HFS) and zero if it does not. We also use $\sigma_{\mathrm{lfs}}$ to identify if the particle is exploring the low field side (LFS). Finally, we define the bounce parameter $\sigma_{b}$ that is set to one if the sign of $v_{\|}$changes during the orbit and zero otherwise. The resulting classification is summarized in the table 1 .

\section{Redistribution of the ions during the sawtooth collapse}

\subsection{Adherence to the perturbation}

An illustration of the redistribution in effective radial position of the thermal helium is given in figure 6 . These particles 


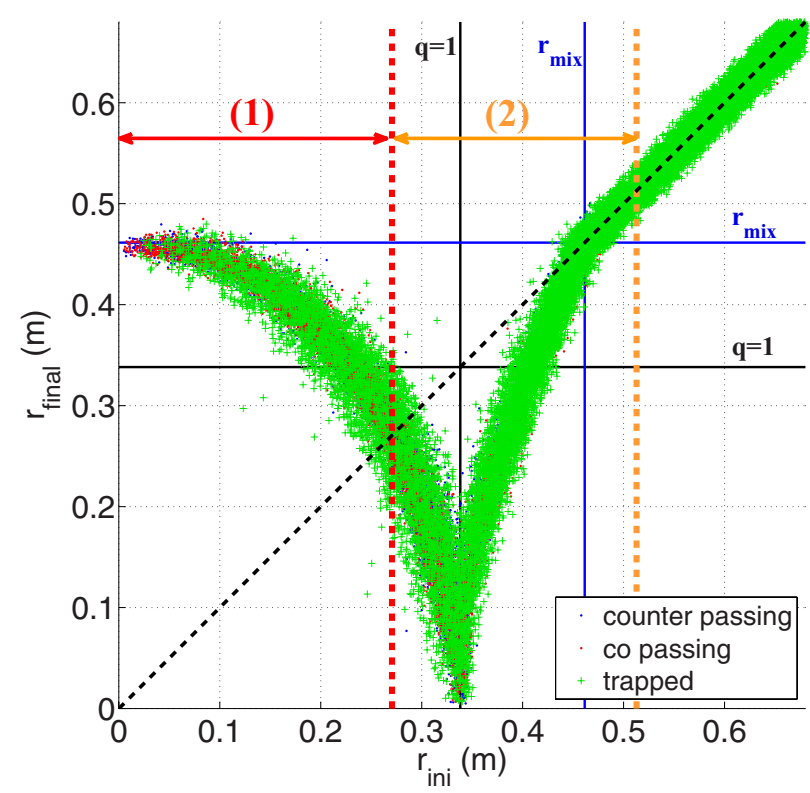

Figure 6. Illustration of the redistribution in effective radial position of the thermal helium. The numbers identify two zones of the plasma: the core (region 1) and the volume enclosing $q=1$ and the mixing radius region (region 2 ). The spread observed in radial position is due to the finite orbit width $\delta r$ of the ions. This is why we use $p_{\varphi}$ as a flux surface label for ions of higher energies.

are almost frozen in the field and follow the reconnection pattern. We may consider two main regions for studying the redistribution: the core (1) from which particles are expelled and the region surrounding $q=1$ and the mixing radius (region 2) where particles are pushed inward.

As was done in [7], we may write the motion of a particle in the helical direction $(\omega=\theta-\varphi)$ as the average of the contribution of three components, related to the electrostatic drift, the longitudinal motion and the 'curvature and gradB' $\operatorname{drift}\left(\boldsymbol{v}_{\mathrm{D}}\right)$ :

$$
\langle\dot{\omega}\rangle=\omega_{v_{E}}+\omega_{\psi}+\omega_{v_{D}} .
$$

The study of $\omega_{\psi}$ and $\omega_{v_{D}}$ in equilibrium gives us a useful tool to predict the behaviour of an ion during the collapse. As was shown on the left side of figure 4 , when we consider the electromagnetic fields at one instant during the crash, the independent drift helical motion $\omega_{v_{D}}$ may average to zero the radial drift when a particle experiences a fast complete helical turn. We thus compare the drift precession motion against a characteristic frequency of the crash: $\omega_{\mathrm{cr}} \sim \pi / \tau_{\mathrm{cr}}$ (half a turn in the $\omega$ direction being considered enough to detach many particles).

On the other hand, the particles with high $\omega_{\psi}$ will tend to stay 'attached' to field lines (or $\psi_{*}$ contours) during the reconnection. A particle may escape the reconnection only if $\omega_{v_{D}}>\omega_{\text {cr }}$ and if $\left|\omega_{v_{D}}\right| /\left|\omega_{\psi}\right|$ is high enough. We evaluate the values of precession in the equilibrium by using the following expressions:

$$
\begin{gathered}
\omega_{\psi}=\left\langle\frac{\partial \theta}{\partial X} v_{\| X}+\frac{\partial \theta}{\partial Z} v_{\| Z}-\frac{v_{\| \varphi}}{R_{0}+X}\right\rangle \simeq \frac{\left\langle v_{\|}\right\rangle(q-1)}{R} \\
\omega_{v_{D}}=\left\langle\frac{\partial \theta}{\partial X} v_{D X}+\frac{\partial \theta}{\partial Z} v_{D Z}-\frac{v_{D \varphi}}{R_{0}+X}\right\rangle .
\end{gathered}
$$

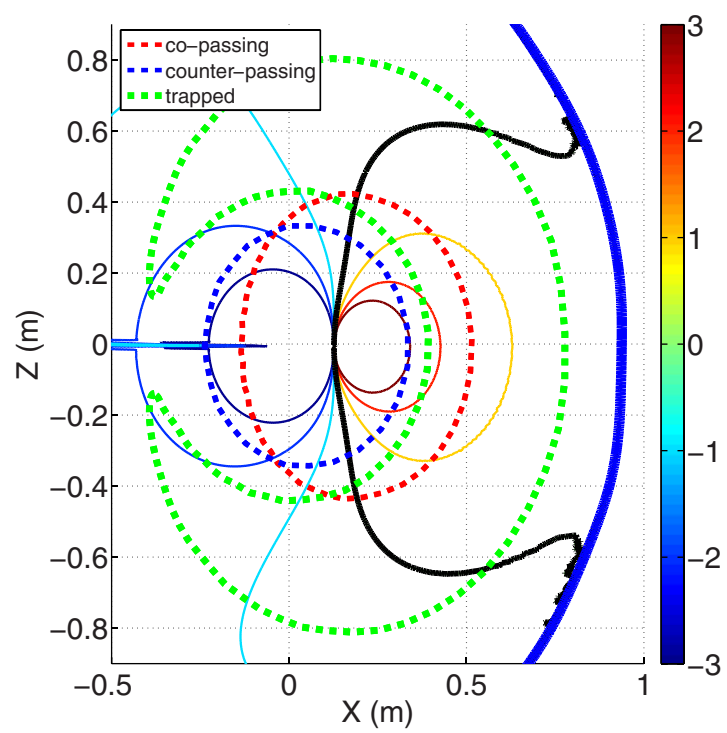

Figure 7. Orbits (dashed lines) in the equilibrium field of $2.2 \mathrm{MeV}$ ions against contours of $\partial \theta / \partial Z$ (thin lines in the background). The thick black line $\partial \theta / \partial Z=0$ separates the positive (LFS) and negative (HFS) regions. Trapped ions with a specific pitch angle $\lambda_{\sigma}$ may spend the same time in the positive gradients as in the negative regions, so that $\omega_{v_{D}} \simeq 0$.

The time that a particle spends in various regions of positive or negative values of $\partial \theta / \partial Z$ will strongly influence $\omega_{v_{D}}$. This is illustrated on figure 7. As was shown in [7], $\omega_{\psi}$ will mostly increase with the distance from the helicity of the resonant surface $|q-1|$. Another effect is the orbit width of the particles. Large values of $\delta r$ will reduce $\omega_{v_{E}}$ (thus reducing the effect of the crash, see figure 4).

In this section, simulations have been performed with an initial uniform density distribution of fast helium. We have studied populations of identical kinetic energy and the spread in pitch angle was given by a uniform distribution in parallel velocity.

\subsection{Behaviour of trapped energetic ions}

For trapped particles, all three terms in equation (10) are significant and of similar magnitudes. The main result of the previous work of Kolesnichenko et al ([7], [13] and [14]) is that, when equaling the crash time with half the drift precession period, a critical energy threshold is obtained:

$$
\mathcal{E}_{\mathrm{crit}} \simeq r_{1} R_{0}\left(Z_{\mathrm{i}} e\right) B_{0} \omega_{\mathrm{cr}} ; \quad \omega_{\mathrm{cr}}=\pi / \tau_{\mathrm{cr}}
$$

This roughly corresponds to $150 \mathrm{keV}$ (for $\tau_{\mathrm{cr}} \sim 144 \mu \mathrm{s}$ ) in our simulations and we may define it from our simulations as the energy beyond which more than half of the trapped particles are not redistributed. Experimental evidence of this threshold may be found in [16].

To quantify in simulations the relative change of flux surface, we evaluate the quantity $\Delta p_{\varphi}=p_{\varphi}$ final $-p_{\varphi}$ initial in regions (1) and (2). Our numerical simulations give us the plot shown on top of figure 8 (in arbitrary units). The particles which have an energy below $\mathcal{E}_{\text {crit }}$ can be considered as 'flux-surface-attached particles'. A clear detachment from the sawtooth of a large majority of the trapped particles is 

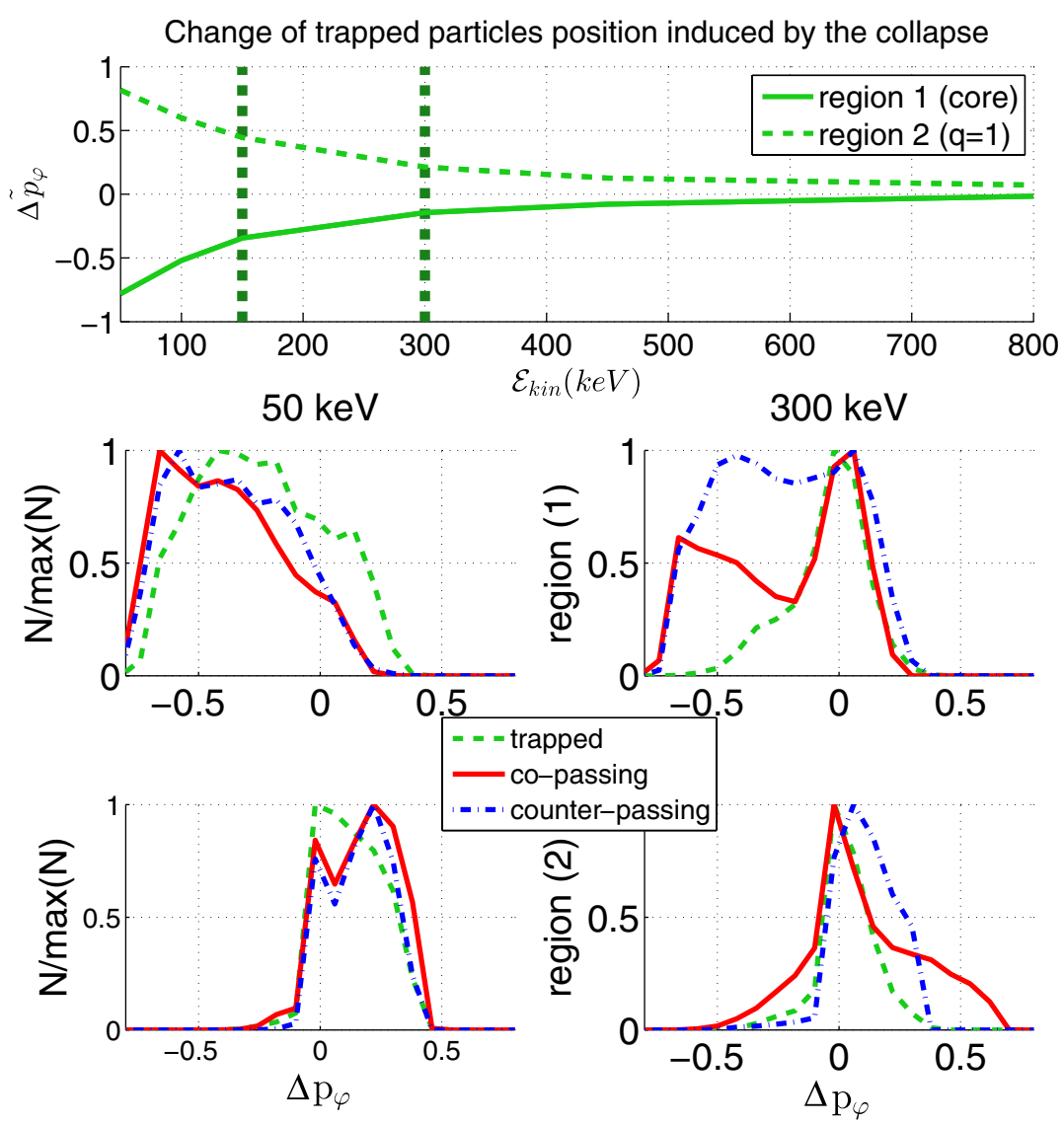

Figure 8. Upper long plot: evolution of $\tilde{\Delta p_{\varphi}}=\Delta p_{\varphi} / \Delta p_{\varphi}\left(\mathcal{E}_{\text {thermal }}\right)$ with average kinetic energy. $\Delta p_{\varphi}\left(\mathcal{E}_{\text {thermal }}\right)$ is the change of $p_{\varphi}$ for the thermal helium. The values are thus normalized to the effect on the thermal populations. We have distinguished ions initially in region (1) and in region (2). The dashed line marks $\mathcal{E}_{\text {crit }}$ (beyond which less than half of trapped particles are redistributed) and $2 \mathcal{E}_{\text {crit }}$. Four lower plots: compared redistribution in $p_{\varphi}$ of helium at $50 \mathrm{keV}$ (left) and $300 \mathrm{keV}$ (right). The top ones show change in $p_{\varphi}$ for region (1), the bottom for region (2). The results are grouped by $\Delta p_{\varphi}$.

seen by allowing them to do a complete helical turn in the $\omega$ direction because of their drift precession: the threshold is then $2 \mathcal{E}_{\text {crit }}(300 \mathrm{keV})$ with our convention. Between $\mathcal{E}_{\text {crit }}$ and $2 \mathcal{E}_{\text {crit }}$, there is a 'transitional' behaviour of the trapped particles, from attached to detached. Numerical simulations indicate that $\mathcal{E}_{\text {kin }}>2 \mathcal{E}_{\text {crit }}$ is more likely to correspond to $\omega_{v D}>\omega_{\text {cr }}$ (figure 9) for most pitch angle values. The change of radial redistribution with energy is detailed for all populations on the lower graphs of figure 8 .

As illustrated on figure 7, when exploring regions of positive and negative $\partial \theta / \partial Z$, a trapped particle may feel on average no precession drift. There is thus evidence of a small fraction of velocity space that has $\omega_{v_{D}} \sim 0$ for a specific pitch angle, $\lambda_{\sigma}$ (figure 9). For intermediate energies in the range $\mathcal{E}_{\text {crit }}$ to $4 \mathcal{E}_{\text {crit }}$, this implies that this very specific population will be redistributed. When increasing energy beyond $4 \mathcal{E}_{\text {crit }}$, we finally observe the detachment of almost all trapped particles. This is related to their large orbit width: we have indeed seen previously that the attachment to the perturbed field is reduced when $\delta r$ gets as large as more than half the mixing radius (figure 4).

On figure 9, there is also evidence of deeply trapped $\left(\lambda_{0}>1\right)$ inward-redistributed particles of high energies in region (2). These are probably captured from the outside by the sawtooth crash that tends to 'attract' particles in the mixing radius region.

\subsection{Behaviour of passing energetic ions}

The attachment of intermediate energy ( $300 \mathrm{keV})$ passing particles (compared with trapped ones) is clearly seen on the four lower plots of figure 8 . Passing ions of high energies (beyond $\mathcal{E}_{\text {crit }}$ ) have a drift precession $\omega_{v_{D}}$ that is on average higher than that of the trapped particles. However they have a much larger value of longitudinal precession $\omega_{\psi}$. They thus tend to stay attached to the pattern of the reconnecting field lines. Both co- and counter-passing particles feel on average almost exactly the same effect from their parallel motion. When we consider figure 10 , we can estimate that we have a detached behaviour of a majority of the passing ions for energies beyond $\mathcal{E}_{c c} \sim 1.2 \mathrm{MeV}$.

On figure 11 , we notice that the geometry of the field has an impact on the threshold $\mathcal{E}_{c c}$. A difference is seen between the low shear case $\left(\mathcal{E}_{c c} \sim 1.2 \mathrm{MeV}\right)$ and the high shear case $\left(\mathcal{E}_{c c} \sim 2 \mathrm{MeV}\right)$. The threshold may be related to an average ratio of the precessions over the reconnecting region: $\left|\omega_{v_{D}}\right|>0.7\left|\omega_{\psi}\right|$.

As a side note, we also remark that the co-passing population and the counter-passing population do not have exactly the same redistribution pattern. The differentiation between the two populations is however complex and closely related to their initial radial position and depending on the magnetic geometry. In our example, we had a uniform initial 

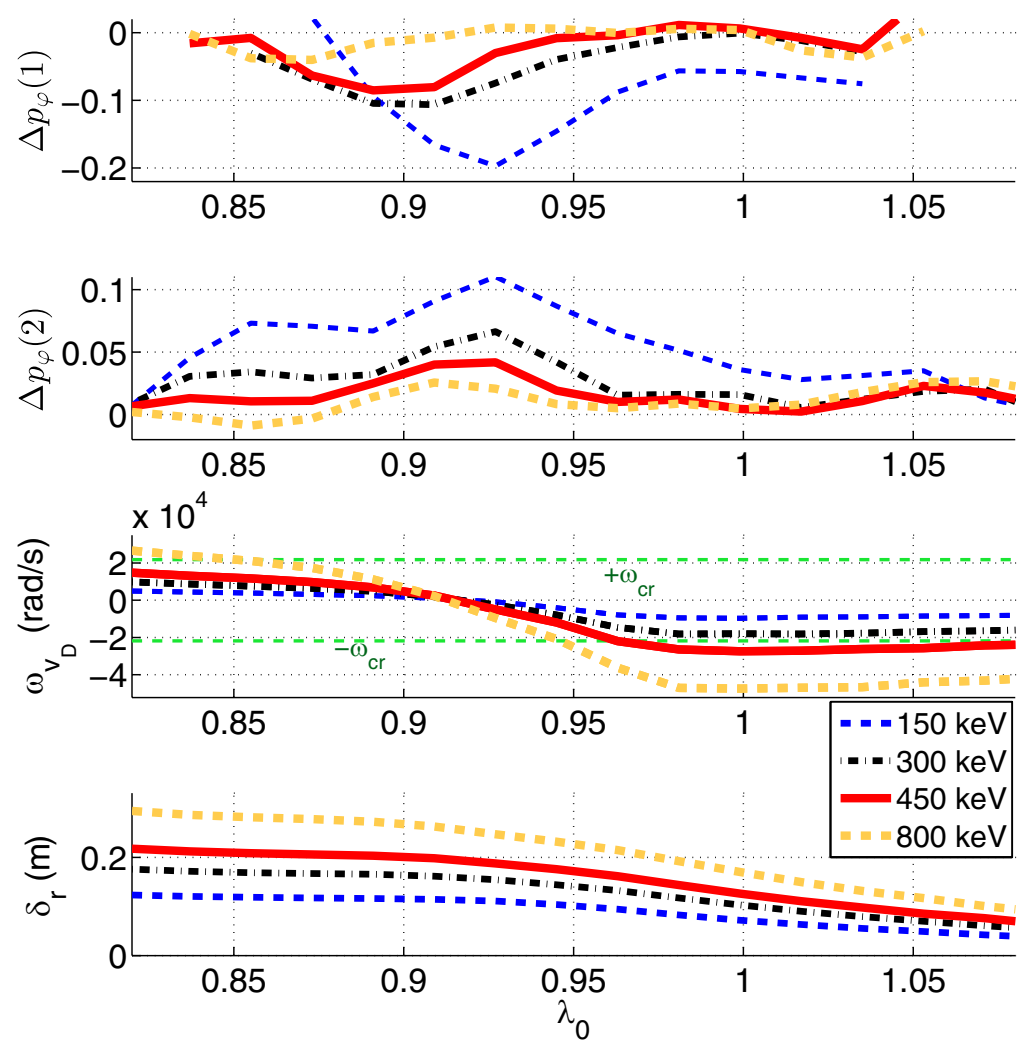

Figure 9. Trapped ions redistribution with respect to their pitch angle $\lambda_{0}$. Trapped ions around $\lambda_{\sigma} \sim 0.92$ may have a helical drift motion close to zero. As a consequence, they stay attached to the perturbation for energies up to $\sim 600 \mathrm{keV}$. This is shown in the two upper graph, where we have differentiated the core (region 1) from the outer (region 2) particles. On the third graph, the two horizontal green lines indicate the values $-\omega_{\mathrm{cr}}$ and $\omega_{\mathrm{cr}}$. The fourth graph gives an estimate of the orbit width of the trapped ions.
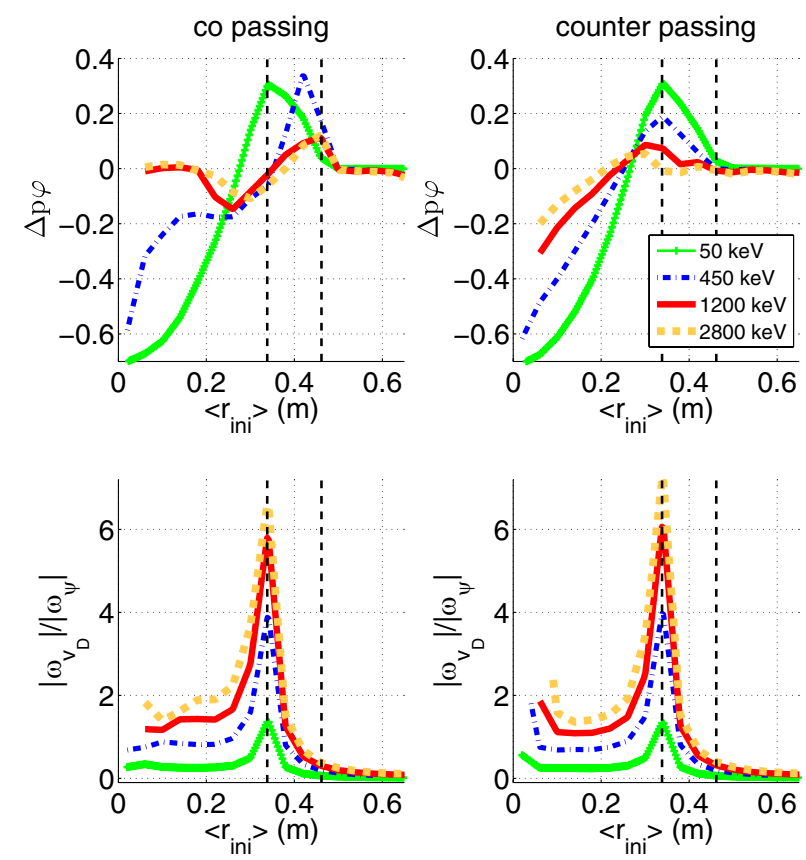

Figure 10. The upper plots shows the change in $p_{\varphi}$ as a function of the orbit-averaged initial radial position of the passing particles. On the lower plots, we represent an estimate of $\left|\omega_{v_{D}}\right| /\left|\omega_{\psi}\right|$. The geometry of the field and of the passing particle orbits has clearly an influence on their redistribution at high energy. On the left is plotted the redistribution of the co-passing and on the right that of the counter-passing. The two vertical dashed lines mark the $q=1$ radial position and the mixing radius position. density of energetic ions over the reconnecting region. The counter-passing ions thus seem to be 'detached' on average more easily when energy increases. However, the main parameter to consider to determine the differentiation of the behaviour of the co- and counter- passing particles is actually their average initial effective radial position (figure 10).

\section{Overall effect of the collapse on a population of fusion-born alphas}

\subsection{A typical equilibrium burning plasma}

In this section, we consider a future hypothetical DT experiment on JET. The alpha-particles produced by fusion reactions have a very high energy $(3.5 \mathrm{MeV})$ compared with the background plasma $(8 \mathrm{keV})$. The energy is transferred from the alphas to the plasma by collisions (first with electrons, then with ions) in about $\tau_{s} \sim 0.5 \mathrm{~s}$. We evaluate the source of fast alphas from fusion as $S_{\alpha} \sim\langle\sigma v\rangle n_{\mathrm{i}}^{2} / 4$ (see [15]). The distribution of alpha-particles is modelled by a localized slowing down distribution according to their energy (see [12]). We denote $\mathcal{E}_{0}$ the birth energy of the alpha-particles (3.5 MeV), $n_{\mathrm{i}}$ the bulk ion density, $m_{\mathrm{i}}$ the average bulk ion mass and we use the distribution functions:

$$
\begin{array}{ll}
f_{\alpha h 0}=\frac{\tau_{s}}{2 \pi} S_{\alpha}\left[\frac{n_{\mathrm{i}}}{n_{\mathrm{e}}}+\left(\frac{v}{v_{c}}\right)^{3}\right]^{-1}, & \mathcal{E} \leqslant \mathcal{E}_{0} \\
f_{\alpha h}=f_{\alpha h 0} \exp \left[\frac{-m_{\alpha}\left(\mathcal{E}-\mathcal{E}_{0}\right)}{T m_{\mathrm{i}}}\right], & \mathcal{E}>\mathcal{E}_{0}
\end{array}
$$



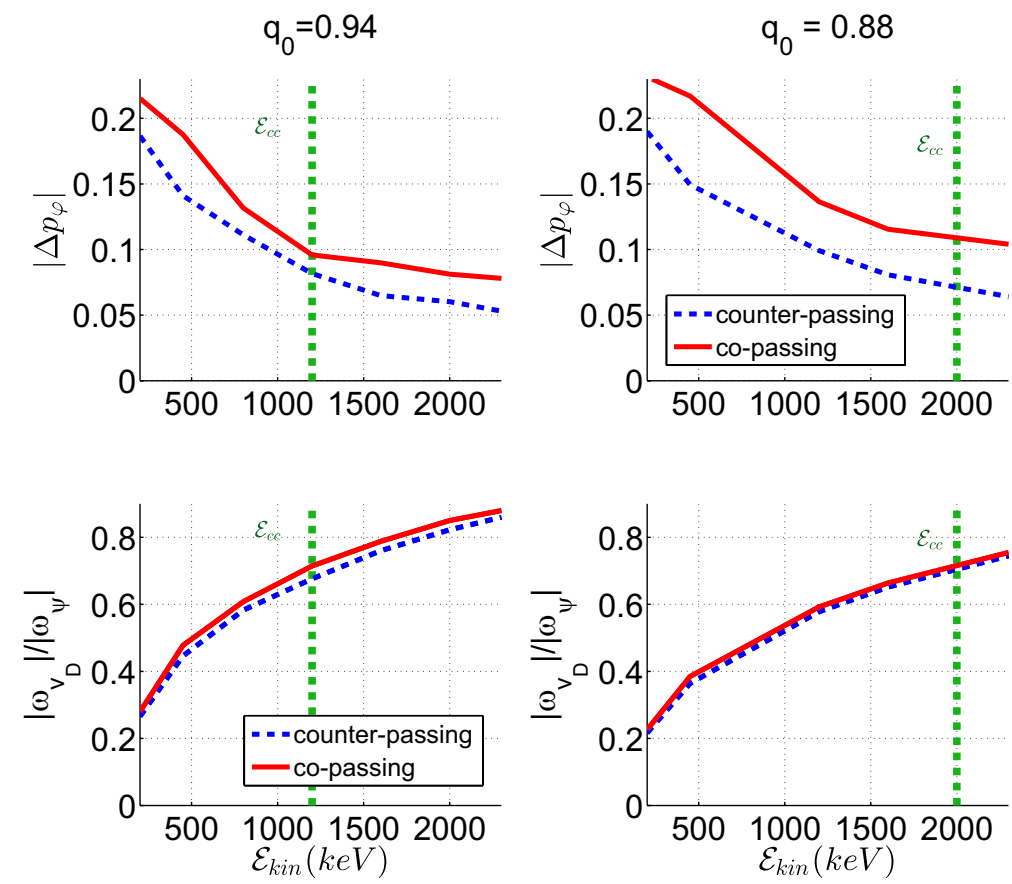

Figure 11. The upper plots shows the change in $p_{\varphi}$ as a function of the average energy of the ions. On the lower plots, we see that passing ions may escape the perturbation for values of $\omega_{v_{D}} / \omega_{\psi}$ sufficiently large. We have marked a threshold at $\mathcal{E}_{\text {kin }}>\mathcal{E}_{c c}$ where the average redistribution of the passing particles has been divided by 2 compared with the thermal behaviour. On average on the entire mixing region, the counter-passing fast ions feel a less pronounced effect of the sawtooth.

where the critical velocity $v_{c}$ is defined as the change of collisional regime for the alphas from electron-dominated $\left(v>v_{c}\right)$ to ion-dominated $\left(v<v_{c}\right)$. We extend the initial spread of alphas to about $2 / 3$ of the radius of the plasma, considering that only these particles will intersect the reconnecting region. In this simplified picture, the alphas are considered to slow down on the average surface on which they are born: any diffusion is neglected. It should be reminded here that the peaking of the density of alphas in the core is what is going to make the difference when considering the global effect of the collapse on the fast ions. In a flat density configuration, there would be almost no overall effect of the collapse on the alpha-particles.

We have done simulations of sawtooth with $\tau_{\mathrm{cr}} \sim 90 \mu \mathrm{s}$. The resulting energy thresholds for detachment of fast helium populations are then approximately $\mathcal{E}_{\text {crit }} \sim 240 \mathrm{keV}$ for the trapped alphas and $\mathcal{E}_{c c} \sim 1.5 \mathrm{MeV}$ for the passing particles (low shear case, $q_{0} \sim 0.94$ ). The source of alphas $S_{\alpha}$ was rescaled to have similar peaking in both equilibria considered.

\subsection{Separation of populations for ions of higher energies}

Simulations confirm detachment of the trapped alpha-particles at energies beyond $\mathcal{E}_{\text {crit }}$ and of a majority of passing alphaparticles at energies beyond $\mathcal{E}_{c c}$ : this is illustrated on figure 12 for the low shear case.

Figure 13 shows the change of density of alpha-particles before and after the collapse for the case of both a low and a higher shear equilibrium. The increase of density in highenergy co- and counter-passing alphas occurs at distinct radial locations (on the outer and inner side of the initial $q=1$ surface, respectively). As was shown in our analysis in the previous section, the amplitude of $\langle|q-1|\rangle$ or $\langle\mathrm{d} q / \mathrm{d} r\rangle r_{\text {mix }}$ (averaging over the reconnecting region) is an important parameter to quantify the behaviour of the passing alphaparticles. Low values of this parameter result in the detachment of passing fast ion: comparing low and higher shear equilibria, we observe a moderate difference (about a factor 2) in the increase of the density around the mixing radius. However, this may be decisive when deriving a post-collapse stability analysis. In figure 13 we can also see that the greater strength of the crash in the higher shear case (second row, right) is reflected in a larger inward transport of trapped particles.

Considering the global efficiency of the crash in terms of helium removal and fast particles confinement, the overall population below $\mathcal{E}_{\text {crit }}$ is about halved in the core, while the high energy population is maintained at higher levels. This can be seen on the upper part of figure 13 where we can assume that the helium ash will follow the bulk of the plasma. The magnetic shear in the core and around the $q=1$ flux surface will be of importance to predict the redistribution of the fast helium. In the usual case of a decreasing density of the passing fast alphas from core to edge, a lower shear in the reconnecting region allows for a better confinement during the collapse.

\section{Conclusions}

We have implemented a numerical model to simulate the effect of a sawtooth collapse on a population of high energy ions. This computational tool has been successfully applied to different magnetic configurations with a population of fusionborn alpha-particles.

When particles have high enough drift precession $\omega_{v_{D}}$, they may escape the pattern of the reconnecting surfaces: there 

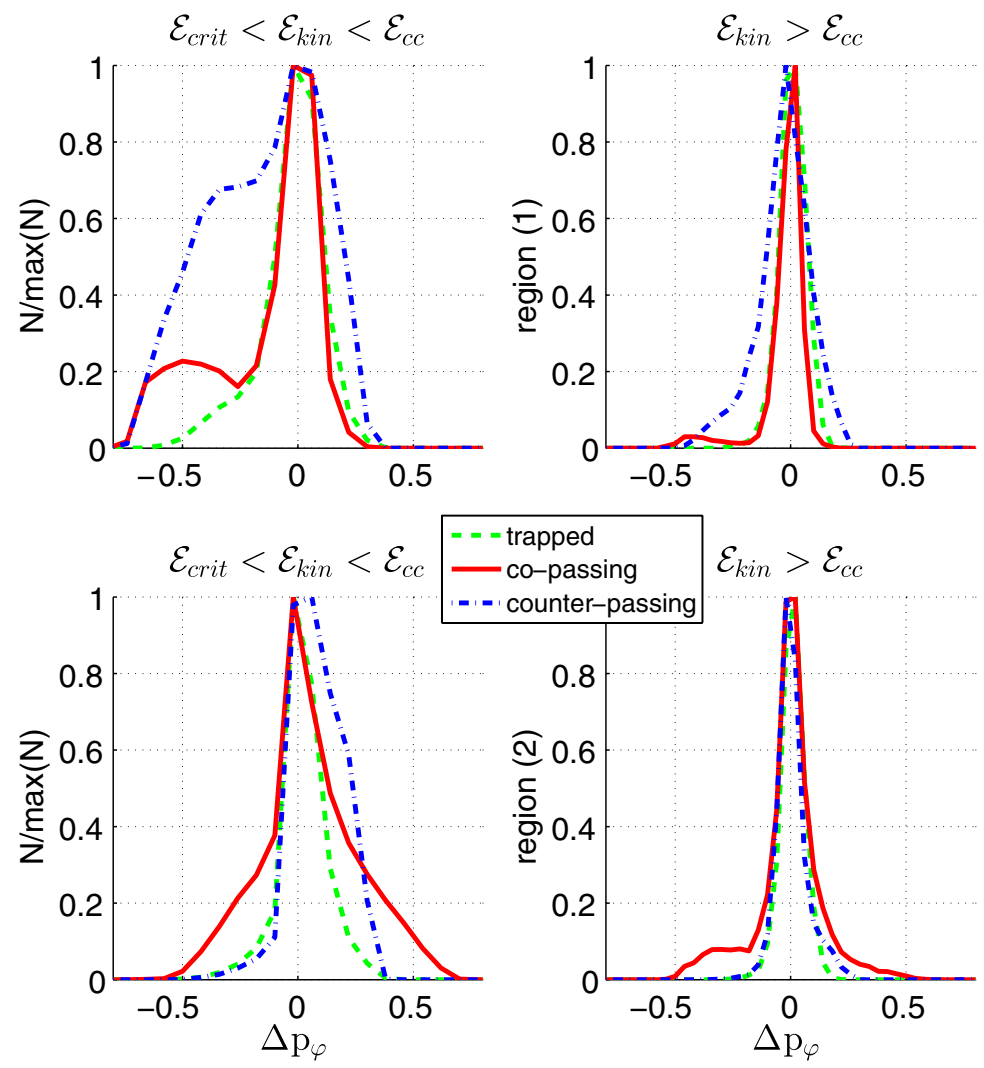

Figure 12. Redistribution of alpha-particles in the low shear case. Top figures: change in $p_{\varphi}$ for alpha-particles initially in the core region (1). Bottom figures: same for the $q=1$ region (2). On the left are gathered $\mathcal{E}_{\text {crit }}<\mathcal{E}_{\text {kin }}<\mathcal{E}_{c c}$ particles, on the right particles beyond $\mathcal{E}_{c c}$.
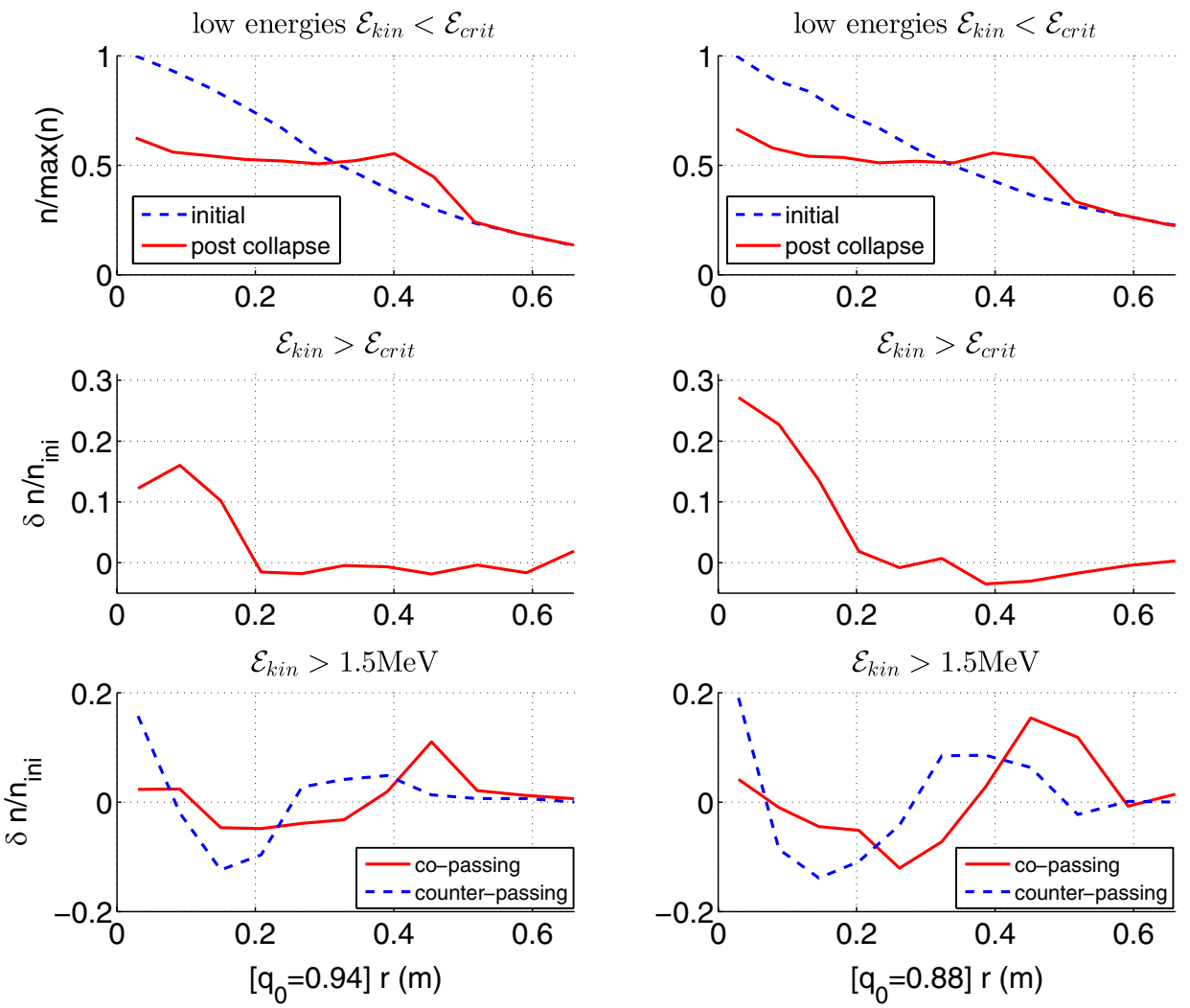

Figure 13. Compared redistributions of alphas between the low shear case $\left(q_{0} \simeq 0.94\right.$ on the left $)$ and the high shear case $\left(q_{0} \simeq 0.88\right.$ on the right). The upper graph illustrates the density redistribution of particles below $\mathcal{E}_{\text {crit }}$. The middle one shows the relative local change of density $\left(\delta n=n_{\text {end }}-n_{\text {ini }}\right)$ for trapped particles beyond $\mathcal{E}_{\text {crit }}$. The lower graph shows the redistribution for passing alpha-particles beyond $\mathcal{E}_{c c} \sim 1.5 \mathrm{MeV}$. To illustrate the effect of the shear the value of $\mathcal{E}_{c c}$ is in both graphs that of the low shear case. 
are thresholds in kinetic energy for 'detachment' of different populations of ions. For trapped ions, we have confirmed the existence of $\mathcal{E}_{\text {crit }}$ for detachment of almost all trapped ions except for a small population around the specific pitch angle $\lambda_{\sigma}$ (that leads to $\omega_{v_{D}} \sim 0$ ). The peculiar behaviour around $\lambda_{\sigma}$ is concealed at intermediate energies due to large orbit width $\delta r$ and fast bounce motion. At higher energies, the large orbit width of the trapped particles mostly prevents their redistribution.

Regarding the passing ions, the threshold $\mathcal{E}_{c c}$ is found at higher energies (beyond a few $\mathcal{E}_{\text {crit }}$ ) and its value depends on the magnetic shear in the core region. Our modelling indicates that, in a low shear configuration, the detachment of the majority of the passing ions occurs progressively over a large range of kinetic energy.

Our study has not addressed reversed shear configurations and the change of orbit category induced by the redistribution. This will be the topic of future studies. Future work will also investigate the induced change on stability of TAEs and NTMs.

\section{Acknowledgments}

The work in this paper has been performed in the framework of the NWO-RFBR Centre of Excellence (grant 047.018.002) on Fusion Physics and Technology. This work, supported by the European Communities under the contract of Association between EURATOM/FOM, was carried out within the framework of the European Fusion Programme. The views and opinions expressed herein do not necessarily reflect those of the European Commission.

\section{References}

[1] Loarte A. et al Progress in the ITER Physics Basis: Chapter 4. Power and particle control Nucl. Fusion 47 S203

[2] Chapman I. et al 2011 Plasma Phys. Control. Fusion 53124003

[3] Porcelli F., Boucher D. and Rosenbluth M.N. 1996 Plasma Phys. Control. Fusion 382163

[4] Graves J.P. et al 2011 Fusion Sci. Technol. 59539

[5] White R.B., Romanelli F. and Bussac M.N. 1990 Phys. Fluids B 2745

[6] Kadomtsev B.B. 1975 Sov. J. Plasma Phys. 1389

[7] Kolesnichenko Ya.I. and Yakovenko Yu.V. 1996 Nucl. Fusion 36159

[8] Beliën A.J.C. et al 2002 J. Comput. Phys. 18291

[9] Wesson J. 2003 Tokamaks 3rd edn (Oxford: Clarendon)

[10] Zakharov L. 1993 Phys. Fluids B 52498

[11] Kolesnichenko Ya.I. et al 1998 Phys. Plasmas 52963

[12] Hazeltime R.D. and Meiss J.D. 2003 Plasma Confinement (New York: Dover)

[13] Kolesnichenko Ya.I. and Yakovenko Yu.V. 1997 Phys. Plasmas 42544

[14] Kolesnichenko Ya.I. and Yakovenko Yu.V. 2000 Nucl. Fusion 401325

[15] Freidberg J. 2007 Plasma Physics and Fusion Energy (Cambridge: Cambridge University Press)

[16] Muscatello C.M. et al 2012 Plasma Phys. Control. Fusion 54025006 